\title{
Kinematic Performance Analysis of a Controllable Mechanism Welding Robot with Joint Clearance
}

\author{
Junjie Gong ${ }^{1}$, Wei Wei ${ }^{1,2}$, Ganwei Cai ${ }^{1 *}$, Yixin Liu ${ }^{1}$ and Sixu Peng ${ }^{1}$ \\ ${ }^{1}$ School of Mechanical Engineering, Guangxi University, Nanning, China \\ ${ }^{2}$ School of Mechanical and Automotive Engineering, South China University of Technology, Guangzhou, China.
}

\begin{abstract}
Because the motor and reducer of the conventional tandem welding robot are installed at the revolute joint, the robot has large rotational inertia and long residual vibration time. However, due to the limitation of workspace and other reasons, the current parallel robot is not suitable for all series welding robots. Therefore, based on the concept of "multi-degree-of-freedom controllable mechanism", a new controllable mechanism welding robot is proposed in this paper. The driving motor and reducer, which have great influence on the main and branch chain of the welding robot, are installed on the frame. The advantage of this design is that the inertia of the robot mechanism is significantly reduced, and its dynamic performance is improved. The position errors of the end members of the welding robot moving along the circle, as well as the angle errors of each joints under the circle movements are analysed.
\end{abstract}

\section{Introduction}

As a common industrial robot, welding robot is widely used in production practice 1 . Because the driving motor and reducer are installed in the joint of the traditional series welding robot, not only the moment of inertia of the robot is increased, but also the residual vibration is difficult to eliminate. However, the current parallel robot has some problems, such as limited working space and high coupling degree, so it is difficult to replace the traditional series welding robot.

Due to the accumulation of the clearance value of each kinematic pair of the traditional series robot, the position error of end components is large. Somashekhar et al.2 proposed a robot vision measurement system, which is used to obtain the motion error of the end position of the mobile robot.Tsai et al. 3used the equivalent kinematic pair to simulate the degree of freedom caused by the joint clearance, and studied the influence of the clearance error of the kinematic pair on the transmission performance of the mechanism. Shi et al. 4 improved the absolute positioning accuracy of KUKA KR5 arc series industrial robot with joint clearance by using the method of on-line error compensation. Zhou et al. 5improved a hybrid least-squares genetic algorithmbased algorithm, and identified and compensated the precision of HA006 series robot with the clearance of joint.

In this paper, the concept of "multi degree of freedom controllable mechanism"6 is applied to industrial robots, and a welding robot with multi degree of freedom controllable mechanism is proposed7. The basic idea of this new type of multi DOF welding robot is to move the motor and reducer installed in the motion joint of the serial robot to the frame as much as possible. The motor is installed on the frame to drive the motion chain, so as to realize the movement of the robot. In this way, the inertia of the robot can be obviously reduced and the time of residual vibration can be shortened. However, the kinematic performance of this new type of multi-degreeof-freedom robot has not been analyzed and studied, especially the influence of its motion pair clearance on its end trajectory. Therefore, based on the clearance model of the continuous contact kinematic pair8, the D$\mathrm{H}$ model9 with clearance of the main kinematic chain of the welding robot is established, and the position error of the end member and the angle error of each joint are analyzed when there is joint clearance under the arc trajectory of the welding robot, which lays the foundation for the follow-up research of the controllable mechanism welding robot.

\section{Structure of welding robot with controllable mechanism}

The controllable mechanism welding robot transfers part of the joint drive motor and reducer to the base, reduces the moment of inertia and residual vibration of the robot, and improves the dynamic performance of the robot. The structure of controllable mechanism welding robot is shown in Figure 1.

\footnotetext{
* Corresponding author: caiganwei@163.com
} 


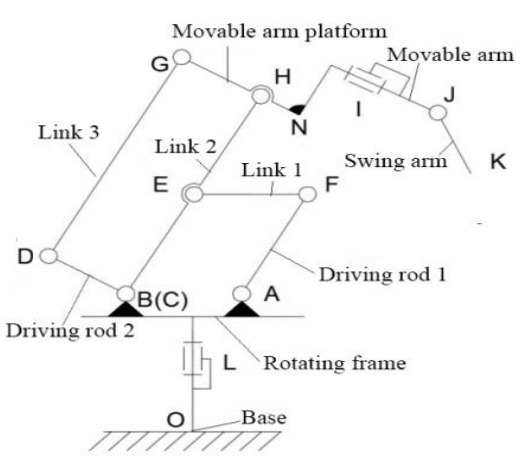

(a)

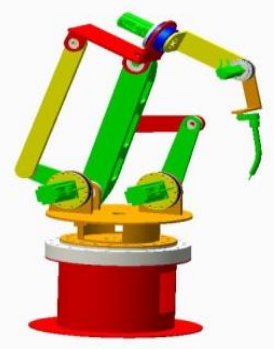

(b)

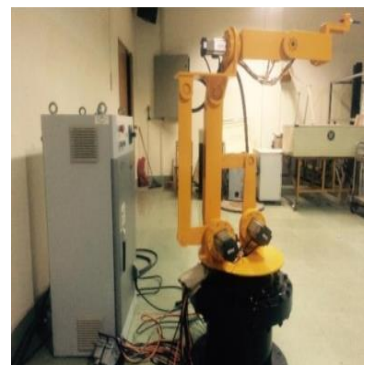

(c)
Figure 1(a) Mechanism diagram of welding robot. (b)A threedimensional picture of a welding robot. (c)The physical prototype of the welding robot.

\section{Kinematics analysis of welding robot considering joint clearance}

\subsection{Vector model of joint clearance}

Establish the clearance model of the kinematic pair as shown in Figure 2. It is assumed that the holes and shafts of the rotating pair are all standard circles, and the radii are $R_{b}$ and $R_{J}$ respectively. When the clearance is not considered, the ideal length of member $\mathrm{A}$ is $l$, and the ideal angular displacement is $\theta$. When considering the clearance between moving pairs, the actual length of member a is $l^{\prime}$, and the actual angular displacement is $\theta^{\prime}$. The following relationships can be obtained:

$$
\left\{\begin{array}{l}
l^{\prime}=\sqrt{(l+r \cos \varphi)^{2}+(r \sin \varphi)^{2}} \\
\theta^{\prime}=\theta+\arcsin \frac{r \sin \varphi}{\sqrt{(l+r \cos \varphi)^{2}+(r \sin \varphi)^{2}}}
\end{array}\right.
$$

According to the actual working track of the controllable welding robot studied in this paper, the clearance model of the moving pair can be regarded as a continuous contact model, and the clearance of the moving pair can be regarded as a certain length of connecting rod without mass. According to the error model:

$$
r=R_{B}-R_{J}, \varphi \in[0,2 \pi]
$$

\subsection{Forward kinematics solution of welding robot considering joint clearance}

Each component of the welding robot is connected by a moving pair. This paper only studies the influence of joint clearances 2 and 3 on the robot end position. The reasons are as follows: a. the joints 1 and 2 are located on the main chain of motion, and the main motion of the whole robot is completed by the main chain of motion; $\mathrm{B}$ the joints 2 and 3 are far away from the end components of the robot, where the clearance has a greater impact on the end through the accumulation of the chain of motion. Because the mechanism welding robot is a complex structure of series parallel combination, its clearance model can be regarded as a continuous contact model to simplify the analysis. Based on this, the kinematics model of welding robot with clearance is established, as shown in Figure 3.

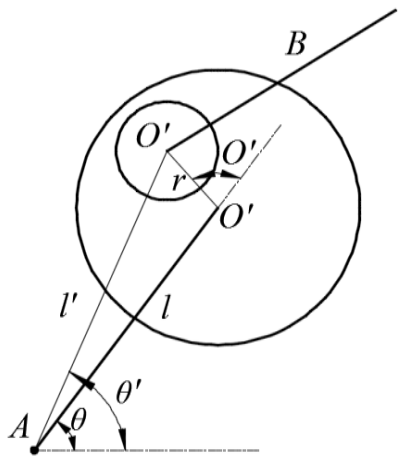

Figure 2 Model of joint clearance

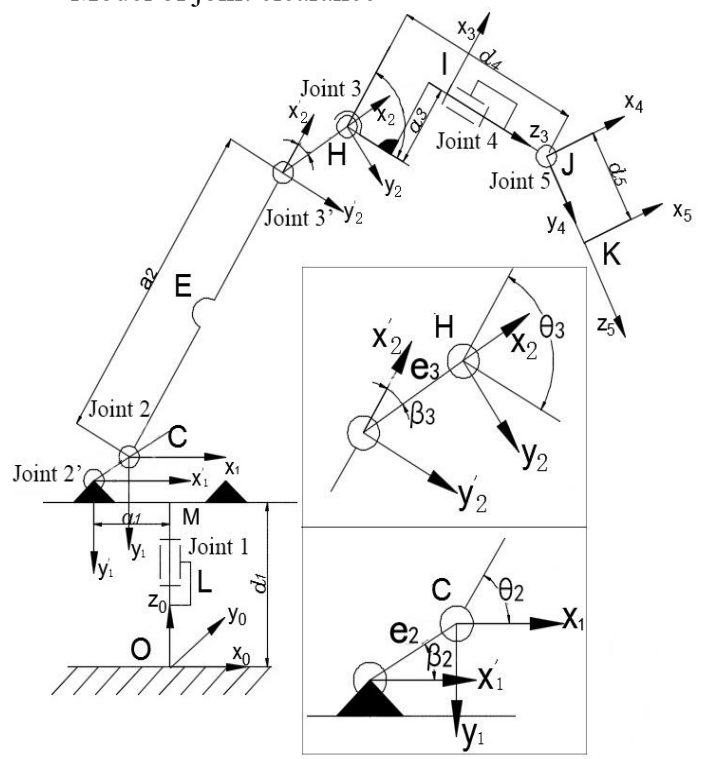

Figure 3 Controllable mechanism welding robot model with joint clearance

The synthetic transformation matrix of robot end is

${ }_{5}^{0} T=\prod_{j=1}^{5}\left({ }_{j}^{j-1} T\left(\theta_{j-1}\right)\right)={ }_{1}^{0} T \cdot{ }_{2}^{1} T \cdot{ }_{3}^{2} T \cdot{ }_{4}^{3} T \cdot{ }_{5}^{4} T$

According to formula(3), the position transformation matrix of welding robot with clearance joint is as follows ${ }_{5}^{0} T^{\prime}=\left[\begin{array}{cc}R_{3 \times 3} & P \\ 000 & 1\end{array}\right]={ }_{1}^{0} T_{2}^{1} T_{2}^{2} T_{3}^{2} T_{3}^{3{ }_{3}^{\prime}} T_{4}^{3} T_{5}^{4} T=\left[\begin{array}{cccc}n_{x}^{\prime} & o_{x}^{\prime} & a_{x}^{\prime} & p_{x}^{\prime} \\ n_{y}^{\prime} & o_{y}^{\prime} & a_{y}^{\prime} & p_{y}^{\prime} \\ n_{z}^{\prime} & o_{z}^{\prime} & a_{z}^{\prime} & p_{z}^{\prime} \\ 0 & 0 & 0 & 1\end{array}\right]$ 
Where, $p_{i}^{\prime}$ is the position matrix of the end actuator of the welding robot with clearance in the base coordinate; $\left[\begin{array}{lll}n_{i}^{\prime} & o_{i}^{\prime} & a_{i}^{\prime}\end{array}\right]$ is the position matrix of the end actuator in the base coordinate.

\section{Analysis of the position error of the end component of welding robot}

\subsection{Analysis of motion track and joint angle}

The arc trajectory of the welding robot is simulated, and the trajectory equation is

$$
x_{0}=68-30 \sin (0.5 \pi t), y_{0}=0, z_{0}=85-30 \cos (0.5 \pi t)
$$

Substituting Eq. (5) into Eq.(4), draw the arc track with Matlab, and then get the speed change of each joint of the clearance welding robot and the ideal welding robot, as shown in Fig. 4.

The arc track motion of welding robot is analyzed. In an ideal state, the trajectory of the robot's end and the angle of its moving joint change smoothly. When considering the clearance, the angle of the moving joint fluctuates slightly. Therefore, in the actual process, due to the reasonable setting of the clearance size of the motion pairs.

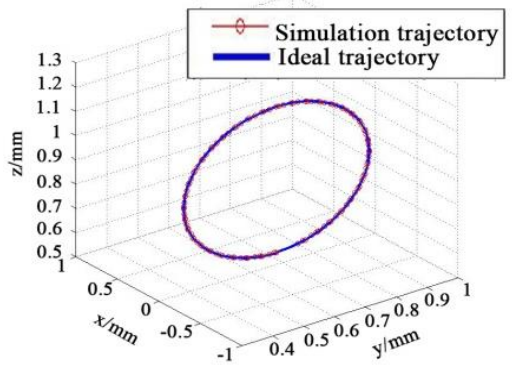

(a)

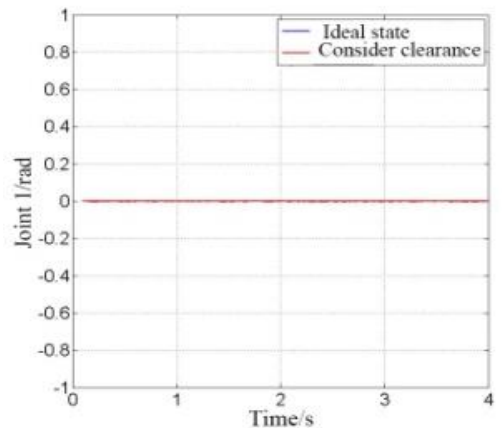

(b)

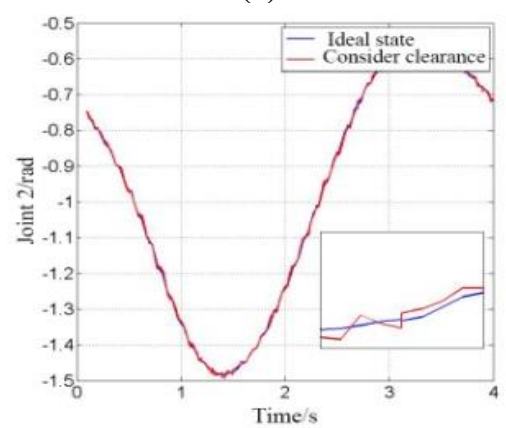

(c)

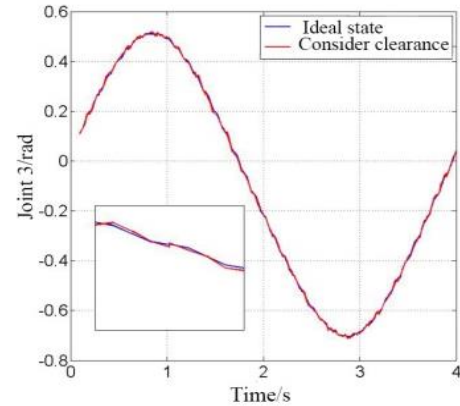

(d)

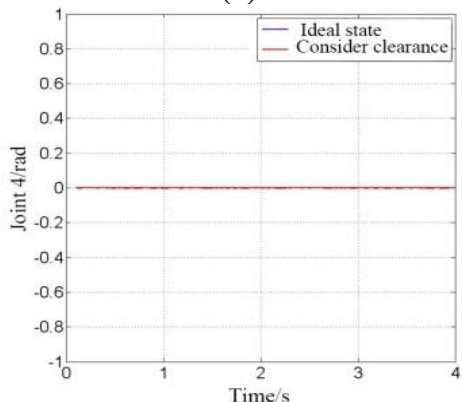

(e)

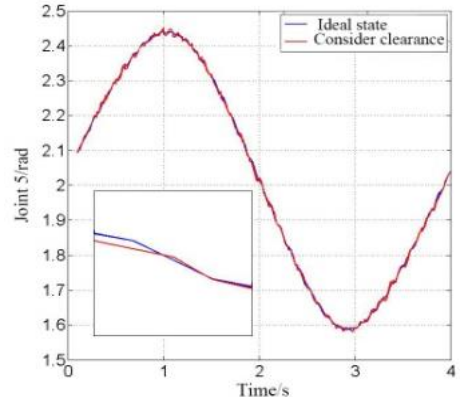

(f)

Figure 4 (a) Arc trajectory simulation of welding robot. (b)-(f) Angle error of joint 1-joint 5

\subsection{Analysis of position error of end component}

According to the kinematic analysis of the welding robot, the difference between the positive kinematics of the ideal model and that of the joint clearance model can be obtained

$\left\{\begin{array}{l}\Delta_{x}=e_{2} c \beta_{2} c \theta_{1}+e_{3} c\left(\beta_{3}+\theta_{2}\right) c \theta_{1} \\ \Delta_{y}=e_{2} c \beta_{2} s \theta_{1}+e_{3} c\left(\beta_{3}+\theta_{2}\right) s \theta_{1} \\ \Delta_{z}=-e_{2} s \beta_{2}-e_{3} c\left(\theta_{2}+\theta_{3}\right)\end{array}\right.$

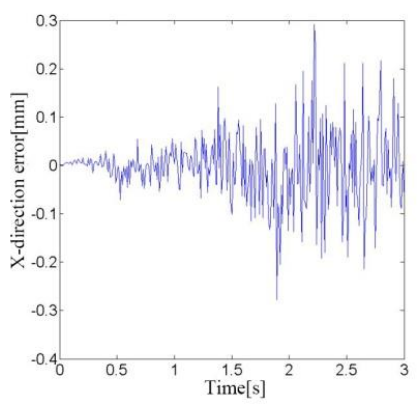

Figure 5 X-direction positio-nal value error of end compon-ent 


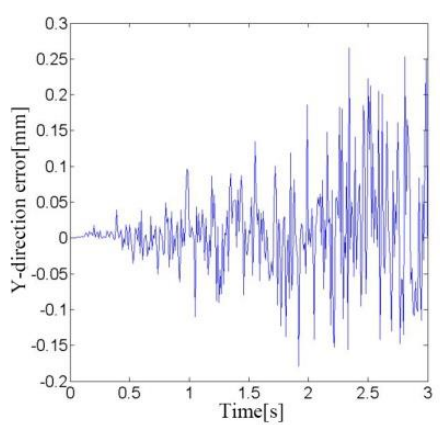

Figure 6 Y-direction positio-nal value error of end compon-ent

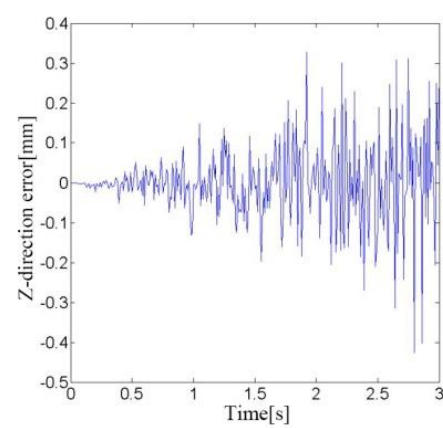

Figure 7 Z-direction posi-tional value error of end co-mponent

It can be seen from equation (6) that the position deviation of the robot's end and the eccentricity angles $\beta_{2}, \beta_{3}$ and the eccentricity distances $e_{2}$ and $e_{3}$ are positive cosine relations, which are difficult to measure and are in chaotic motion. Therefore, it can be taken that the eccentricity angle obeys the uniform distribution $U(0,2 \pi)$ and the eccentricity obeys the normal distribution $N(0.1,0.1)$. Set the clearance value to $0.1 \mathrm{~mm}$, in 3 seconds, $\theta_{1}$ from $0^{\circ}$ to $60^{\circ}, \theta_{2}$ and $\theta_{3}$ from $-60^{\circ}$ to $0^{\circ}$. The variation rule of robot end component error is shown in Figure 5 to Figure 7.

According to figure 8 , when the clearance value is $0.1 \mathrm{~mm}$, the error range of the robot in $\mathrm{X}$ direction is $(0$ $0.3 \mathrm{~mm})$, in $\mathrm{Y}$ direction is $(0-0.25 \mathrm{~mm})$, and in $\mathrm{Z}$ direction is $(0-0.5 \mathrm{~mm})$. It can be seen that when the clearance value is $0.1 \mathrm{~mm}$, the maximum error of the end position is $0.5 \mathrm{~mm}$, which has a great influence on the quality of the welding machine required for high-precision welding. Therefore, it is the key to design the welding robot to set the allowable clearance value reasonably.

\section{Conclusion}

In this paper, a new type of multi-DOF robot mechanism is proposed. The part of motors and reducers that were originally installed in the motion joints are installed on the frame, which reduces the inertia of the robot and improves the dynamic performance of the robot. In this paper, its kinematics performance is studied. The D-H method is used to establish the kinematic model of the welding robot with clearance. Based on the clearance model of continuous contact, the angle variation relationship of each joint of the welding robot with clearance is analyzed. When the clearance value is $0.1 \mathrm{~mm}$, the range of end position error of welding robot is obtained.

The author(s) disclosed receipt of the following financial support for the research, authorship, and/or publication of this article. The work was supported by National Natural Science Foundation of China Grant No. 51765005.

\section{References}

1. Haitao L, Jia F, Lichuang J, Guangming L, Chuangshuai Y, and Tingke W. Kinematics and dynamics analysis of a new-type friction stir welding robot and its simulation. Advances in Mechanical Engineering, vol. 11, pp.1-12, 2019.

2. Somashekhar S Hiremath, Robins Mathew, and Jennifer Jacob. Implementation of Low Cost Vision Based Measurement System: Motion Analysis of Indoor Robot, International Journal of Mechanical Engineering and Robotics Research, Vol. 7(6), pp. 575-582, 2018.

3. Tsai M J, Lai T H. Kinematic sensitivity analysis of linkage with joint clearance based on transmission quality. Mechanism and Machine Theory,vol. 39,pp.1189-1206,2004.

4. Xiaojia S, Fumin Z, Xinghua Q, Bailing L, and Junlong W. Position and Attitude Measurement and Online Errors Compensation for KUKA Industrial Robots. Journal of Mechanical Engineering, 2017, 53(8): 1-7.

5. Jian Z, Heejun K. A hybrid least-squares genetic algorithm-based algorithm for simultaneous identification of geometric and compliance errors in industrial robots. Advances in Mechanical Engineering, vol. 7(6),pp.1-12,2015.

6. Ganwei C, Yuchen P, Hongzhou W, and Jiansheng D. Functional Analysis Based Type Synthesis of a Novel Type of Loading Mechanisms. Journal of Mechanical Engineering.vol. 50,pp.50-59,2014.

7. Ganwei C, Mingyu D, Huali R, Jianbo T, Chengliang W, Honghan L, and Bin Y. Fivedegree-of-freedom series-parallel controllable mechanism welding robot, China, Patent, 201610464088.1 [P].2016-06-23.

8. Flores P, Ambrosio J, Claro JCP, Lankarani HM, Koshy CS. A study on dynamics of mechanical systems including joints with clearance and lubrication. Mechanism \& Machine Theory, vol, 41, pp. 247-261, 2006.

9. $\mathrm{Xu} \mathrm{Z,} \mathrm{Zelong} \mathrm{Z,} \mathrm{Yong} \mathrm{Q.} \mathrm{Parameter} \mathrm{Identification}$ and Calibration of D-H Model for 6-DOF Serial Robots. ROBOT, vol, 38,pp. 360-370, 2016. 